\title{
Vergleich der französischen und englischen Übersetzungen von Hölderlins Elegien: Von der heideggerianischen Wörtlichkeit zur Neuschöpfung
}

\begin{abstract}
Die Übersetzung von Hölderlin bereitet Schwierigkeiten, einerseits wegen der schwierigen Sprache des deutschen Dichters und andererseits wegen des durch seine chaotische Pindarsche Sprache geäußerten komplexen geistigen Inhalts. Angesichts der Unterschiede zwischen beiden Sprachen bringt der Vergleich der französischen und englischen Übersetzungen verschiedene Strategien zur Geltung: Die französischen heideggerianischen „Jünger der Wörtlichkeit“ versuchen, die chaotische Sprache des Dichters und so dessen subtile Philosophie wiederzugeben. Allerdings weisen ihre Übersetzungen hier und da Inkorrektheiten auf, die der deutsche Text nicht beinhaltet. Die dem Deutschen näher stehende englische Sprache wiederum erlaubt es den Übersetzern, Hölderlins Syntax und Verse getreuer wiederzugeben. Ansonsten eint die französischsprachigen schweizerischen Übersetzer, die sich von den „Jüngern der Wörtlichkeit“ durch die Wahl einer schlichteren und korrekteren Sprache unterscheiden und dadurch eine angenehmere Übersetzung liefern, und die englischen Dichter eine gemeinsame Tendenz, die esoterisch anmutenden Verse Hölderlins pädagogisch zu explizieren und so die verzwickte Pindarsche Sprache zu lockern. Schließlich bevorzugen die schweizerischen und englischen Übersetzer-Dichter eine fruchtbringende Nachdichtung, die eine gewisse Osmose zwischen dem übersetzten Werk und dem Werk des übersetzenden Dichters erahnen lässt.
\end{abstract}

Keywords: esoterische Übersetzung, explizierende Übersetzung, Nachdichtung, philosophische Dichtung, wörtliche Übersetzung

Viele große Dichter haben fremde Texte in die eigene Sprache übersetzt, um am eigenen Stil zu feilen. Zu den am häufigsten ins Französische und Englische übersetzten deutschen Dichtern gehört Hölderlin. Dieser hat sich selbst dem Übersetzen gewidmet und wurde wiederum in beiden Sprachen von Dichtern übersetzt. So nahm ich mir vor, die Übersetzungen seiner Dichtung ins Französische und ins Englische zu vergleichen. Hölderlins Werk erweist sich als umfangreich. Daher konzentrierte ich mich auf seine Elegien, die einen zentralen Platz in seinem Werk einnehmen und ein förmlich und inhaltlich homogenes Ganzes bilden. Hölderlin 
trauert darin um seine verstorbene Diotima, den vergangenen Ruhm Griechenlands und den Abschied der Götter von der sterblichen Welt. Letzteren soll der Dichter als Vermittler zwischen den Menschen und dem Göttlichen kompensieren und dabei in der christlichen Logik der Parusie die Erinnerung an die Götter im Diesseits wachhalten, um deren Rückkehr vorzubereiten.

Die Wahl eines solchen Vergleichs lässt sich zudem einerseits durch die enge Verwandtschaft des Deutschen und des Englischen und andererseits durch den großen Unterschied zwischen dem Französischen und dem Deutschen begründen. Diese Umstände dürften mir erlauben, verschiedene Übersetzungsstrategien herauszuarbeiten. Mit dem Dilemma konfrontiert, sich vom ursprünglichen Sinn zu entfernen, um den Erwartungshorizont der fremdsprachlichen Leser zu befriedigen oder im Gegenteil den Text getreu, aber dafür fremd und rätselhaft zu übertragen, muss der Übersetzer eine schwere Wahl treffen. Im Fall Hölderlins schwanken seine französischen und englischen Übersetzungen zwischen den beiden Polen der wörtlichen Wiedergabe und der Neuschöpfung, zwischen einem esoterischen Stil und einer pädagogischen Interpretationsanstrengung, die sich mithilfe des Standpunkts jener französischen Übersetzungswissenschaftler erforschen lassen, mit denen ich im Rahmen der Histoire de la Traduction en Langue Française arbeiten durfte. Es handelt sich dabei um eine philologische Tradition, die anhand einer stilistischen Studie einschätzt, inwiefern der Übersetzer dem ursprünglichen Text treu geblieben ist und den Erwartungshorizont der Leser befriedigt. So ließe sich fragen, welche theoretische Methode der Übersetzung die Quintessenz von Hölderlins Dichtung am besten wiedergibt. Wenn ich mich zunächst auf die französische Übersetzungsschule von Heideggers ,Jüngern der Wörtlichkeit' konzentriere, so kann ich danach die dichterische Übertragung der Elegien durch französischsprachige und englischsprachige Übersetzer miteinander vergleichen, bevor ich die Osmose zwischen den Übersetzungen und dem Werk der übersetzenden Dichter erforsche.

\section{Die französische Übersetzungsschule von Heideggers ,Jüngern der Wörtlichkeit'}

\subsection{Die Theorie der Wörtlichkeit}

Michel Deguy erwähnt im Mai 1986 in einer Ansprache in Tübingen die französischen Übersetzungen Hölderlins. Er bezieht sich darin auf die Pléiade-Ausgabe (1967), die zwei Generationen von Übersetzern, also Gustave Roud und François Fédier zusammenbringt (Deguy, in Courtine 1989, 134), und preist deren Stil in der 
Tradition der Heidegger-Schule, den „so charakteristischen Ton“ der französischen Übersetzungen von Hölderlin, welcher einer wörtlichen Übersetzung entspricht, die vom Philosophiedozenten Fédier, einem weiteren Jünger der französischen Heidegger-Schule, vertreten wird. Dieser begründet seine Übersetzungsmethode mit Hölderlins eigenen „maximal wörtlichen“ Übersetzungen [,la littéralité maximale“] (Fédier 1989, 10-13), unter denen er keinen einzigen Fehler gefunden habe, und er hoffe, dass seine Übersetzungen niemals dunkel anmuteten. Er expliziert seine Methode folgendermaßen:

Comment traduire maximalement ? [...] il suffit de rendre toutes les particularités transposables, par exemple les anomalies de place des mots, les formes grammaticales inhabituelles, les figures singulières [...], parce que, chez Hölderlin [...], les irrégularités ne sont pas des imperfections, mais des signes. (Fédier, in Courtine 1989, 471)

Die heideggerianische Tradition dieser Übersetzungsschule erklärt Deguy mit Heideggers Ablehnung, bei Hölderlin das Dichterische vom Philosophischen zu trennen, und mit dessen Willen, seiner Dichtung einen philosophischen Sinn zu verleihen: „La violence anti-inductive de Heidegger [...] nous aidait à refuser cette séparation de corps et de biens, j'allais dire ,très française‘, entre littérature et philosophie.“ (Deguy, in Courtine 1989, 133) Deguy bezieht sich hier auf Heideggers Vorworte zu seinen Hölderlin-Kommentaren: „Die Erläuterungen gehören in das Gespräch eines Denkens mit einem Dichten, dessen geschichtliche Einzigkeit niemals literarhistorisch bewiesen, in die jedoch durch das denkende Gespräch gewiesen werden kann.“ (Heidegger 1944, 7)

Der Wille, die Dichtung nicht von der Philosophie zu trennen, rechtfertigt demnach die wörtliche Übersetzung, die grammatische Besonderheiten als Zeichen aufrechterhält. Nicht von ungefähr spielt Deguy in seiner Ansprache von 1986 auf Antoine Bermans Monographie über die Übersetzung als „épreuve de l'étranger“ (Deguy, in Courtine 1989, 131) an. Dieser behauptet nämlich, um die wörtliche Übersetzung zu verteidigen:

Que la traduction qui „sent“ la traduction soit par ailleurs considérée comme mauvaise, c'est là un contresens, qui méconnaît que l'écriture d'une traduction est un mode d'écriture irréductible : une écriture qui accueille dans sa langue propre l'écriture d'une autre langue, et qui ne peut, sous peine d'imposture, faire oublier qu'elle est cette opération. (Berman 1984, 249) 


\title{
1.2 Die Praxis der wörtlichen Übersetzung
}

Fédiers wörtliche Übersetzung wirkt teilweise überraschend, namentlich in der Elegie „Brot und Wein“. Wenn Hölderlin in den Versen 31 bis 33 schreibt:

\begin{abstract}
Aber sie muß uns auch, daß in der zaudernden Weile, Daß im Finstern für uns einiges Haltbare sei

Uns die Vergessenheit und das Heiligtrunkene gönnen.

(Hölderlin 1992, 286)
\end{abstract}

übersetzt Fédier:

Mais il lui faut aussi, à nous, afin que dans le temps qui hésite

Que dans la ténèbre pour nous quelque chose soit tenable,

Nous accorder l'oubli et l'enivrement sacré.

(Fédier, in Courtine 1989, 18)

Hier ahmt Fédier Hölderlins deutschen Satzbau nach. Der deutsche Dichter unterbricht seinen Hauptsatz, um einen mündlichen Stil einzuführen, ohne jedoch dabei gegen grammatische Regeln zu verstoßen. Diese Unterbrechung mutet auf Französisch ungeschickt an, da Fédier - wie im Deutschen - das Personalpronomen « nous » vor die Nebensätze stellt. Das Verb erscheint somit viel später und bleibt vom zugehörigen Personalpronomen abgeschottet, was die französische Grammatik stört.

Außerdem wiederholt Fédier wie Hölderlin die Konjunktion „que“ (,dass“) und leitet so eine stilistische Diskordanz ein, die an grammatische Inkorrektheit grenzt.

Deguy entschuldigt solche Fehler in der Übersetzung mit dem Modell Pindars, von dem sich Hölderlin inspirieren lie $\beta^{1}$ : „Loin qu'il s'agisse d'une ,licence poétique، qui ,se permet' quelques fautes excusables au titre du mètre ou de la signification (par exemple), nous y entendons l'esprit de poésie. [...] Esprit d'audace selon Pindare.“ (Deguy, in Courtine 1989, 135) Es ließe sich allerdings folgender Einwand erheben: Hölderlins Sprache mag selbst einem deutschen Leser noch so verzwickt und gewunden, ja manchmal rätselhaft anmuten, sie bleibt doch grammatisch gesehen korrekt, was in der Übersetzung von Fédier nicht immer der Fall ist.

Schließlich ist auch die Wahl der Wörter näher zu betrachten. Hölderlin benutzt Begriffe, wie beispielsweise den Ausdruck „in der zaudernden Weile“ oder

1 Schmidt 1984, 66: „Klopstock, Goethe und Hölderlin sahen darin [in Pindars freiem Dichten] eine wesentliche Legitimation für die Schaffung großer Gedichte in freien Rhythmen.“ 
das Nominaladjektiv „einiges Haltbare“, die philosophische Tiefe nahelegen. In der Geschichte der deutschen Sprachbildung haben bereits Meister Eckhart und Luther das deutsche Volk an Neubildungen gewöhnt. Da die seit Malherbe und Boileau auf Klarheit bestehende französische Sprache jedoch keine derart flexible Anwendung vorsieht, wirken die Ausdrücke von Fédier „le temps qui hésite“ und „quelque chose de tenable“ nicht sehr verständlich. In seiner Übersetzung vom „Wanderer“ steht ebenfalls:

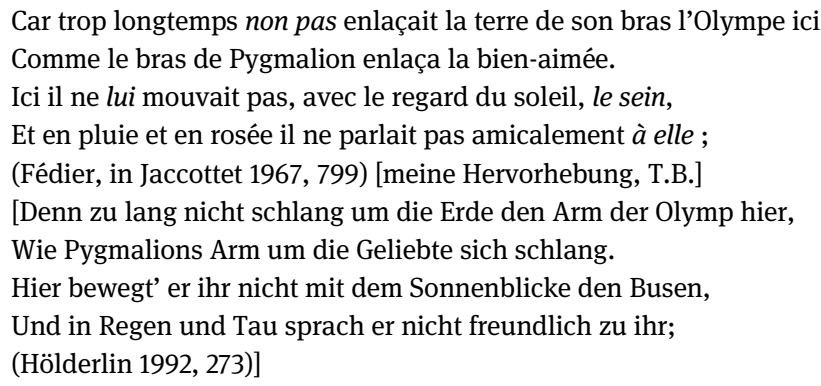

Hier übersetzt Fédier mit hervorgehobenen Germanismen, die eine Nachahmung des deutschen Satzbaus bezwecken. Diese werden der französischen Sprache allerdings nicht angepasst und wirken inkorrekter als auf Deutsch. Insbesondere die Verschiebung des Pronomens „à elle“ ans Ende des Satzes und seine Einführung mit der Präposition „à “ hinterlassen einen fehlerhaften Eindruck. Diese Übersetzungsmethode erinnert an die Polemik, die Fédiers Übersetzung von Heidegger ins Französische auslöste. Robert Maggiori bemerkt sehr kritisch: „L'effort est certes admirable [...]: mais est-ce au prix de la rendre étrangère, sinon d'en faire un sabir?“ (Maggiori 2013)

Die Wörtlichkeit und die damit einhergehende Entstehung von Neubildungen werden als zu fremd empfunden. Die Übersetzung eines philosophischen Textes wird zwar nicht auf dieselbe Weise wie die eines Gedichtes wahrgenommen. Die Polemik gegen Fédiers Übersetzung von Heidegger verdeutlicht jedoch das Unbehagen, das eine solche wörtliche Methode auch im philosophischen Bereich erwecken kann. 


\title{
2 Dichterische Übertragung der Hölderlinschen Dichtung ins Französische und ins Englische
}

\subsection{Die expliziten Übersetzungen der Dichter Jaccottet und Roud}

Hölderlins Dichtung kann durch die Übersetzung von Dichtern anders als durch jene von Philosophen wiedergegeben werden. Der Dichter Philippe Jaccottet widersetzt sich der Dominanz der Philosophen, und insbesondere jener der berühmten Philosophen im Bereich der Poesie. So denunziert Jaccottet die Heideggerianische Schule. In einem Brief, den Jaccottet mir persönlich über dieses Thema schrieb, präzisierte er, er finde Heideggers Hölderlin-Lektüre, und namentlich die seiner Jünger, autoritär, wenn nicht diktatorisch. Jaccottet begründet seine kritische Distanz zu den Heideggerianern anhand der Elegie, in deren Tradition er selbst die Rolle des Dichters als Vermittler zwischen Menschen und Göttern und als Hüter des Lichts (,veilleurs [...] fidèles à l'antique lumière“ [Jaccottet 1987, 302]), bzw. als Hüter der Hoffnung zur Geltung bringt. In dieser Hinsicht zeigt er sich einem zweiten schweizerischen Dichter und Übersetzer von Hölderlin sehr nah, nämlich Gustave Roud, der von der Melancholie der einzigen Elegie, die er übersetzt hatte, fasziniert war.

Die Übersetzungen Hölderlins durch Jaccottet und Roud setzen sich von denen Fédiers und Deguys ab, indem sie sich von einem wörtlichen Vorgehen abwenden und die Einfachheit, die Verständlichkeit und die treue Wiedergabe gleichzeitig fördern. Vergleichen wir eine Übersetzung von „Brot und Wein“ durch Fédier mit einer von Roud. Hölderlin schreibt:

\footnotetext{
Aber sie muß uns auch, daß in der zaudernden Weile,

Daß im Finstern für uns einiges Haltbare sei

Uns die Vergessenheit und das Heiligtrunkene gönnen.

(Hölderlin 1992, 286)
}

Fédier übersetzt:

\begin{abstract}
Mais il lui faut aussi, à nous, afin que le temps qui hésite Que dans la ténèbre pour nous quelque chose soit tenable, Nous accorder l'oubli et l'enivrement sacré.

(Fédier, in Courtine 1989, 18)
\end{abstract}

Roud seinerseits überträgt diese Verse folgendermaßen:

Mais qu'elle aussi (car il le faut afin qu'en notre lent séjour

Dans cette ombre quelque chose nous soit gardé qui nous conforte) 
Qu'elle aussi nous donne l'oubli, qu'elle aussi nous donne l'ivresse

Sacrée.

(Roud, in Jaccottet 1967, 809)

Aus dem Vergleich ergibt sich, dass Roud statt Fédiers wörtlicher Übersetzung Hölderlins esoterische Verse expliziert, wobei er nicht zögert, die eindeutige Formulierung in Klammern zu setzen, um seine pädagogische Geste zu betonen. Die Wiederholung des Adverbs „aussi“ („auch“) dient hier dazu, den Parallelismus hervorzuheben, der aus der eindeutigen Formulierung der bloßen, auf Deutsch mit „und“ eingeführten Nebenordnung entsteht. Das Explizieren erläutert das Rätselhafte an der Nacht, in welcher die Sterblichen das verlorene goldene Zeitalter vergessen und über diesen tragischen Verlust vom Dichter hinweggetröstet werden.

\subsection{Explizierung der Hölderlinschen Verse durch die englischen Übersetzer}

Bei den englischen Übersetzern beobachten wir dieselbe Tendenz, den Text Hölderlins deutlicher auszudrücken und über jede zweideutige Formulierung hinaus ein Missverständnis zu vermeiden. Zu erwähnen ist zunächst die Übersetzung von Michael Hamburger - die erste ins Englische (1952). Seine Übersetzung erweist sich in unserer Untersuchung als interessant, da er selbst deutschsprachig ist und Hölderlin in eine seiner Erstsprache nahestehende Sprache übersetzt. Hamburger neigt dazu, Hölderlins Verse zu explizieren. Diese Neigung geht manchmal so weit, dass die Erläuterung an eine Interpretation grenzt. Schreibt Hölderlin in „Heimkunft“:

Engel des Hauses, kommt in die Adern alle des Lebens,

Alle freuend zugleich, theile das Himmlische sich!

Adle! Verjünge! Damit nichts Menschlichgutes, damit nicht

Eine Stunde des Tags ohne die Frohen und auch

Solche Freude, wie jetzt, wenn Liebende wieder sich finden,

Wie es gehört für sie, schicklich geheiliget sei.

(Hölderlin 1992, 294)

dann übersetzt Hamburger:

Angels, too, of our house, re-enter the veins of all life now, Gladdening all at once, let what is heavenly be shared!

Make us noble and new! Till nothing that's humanly good, no

Hour of the day without them, them the most joyful, or such 
Joy as now too is known when lovers return to each other, Passes, as fitting for them, hallowed as angels demand.

(Hamburger 1966, 165)

Die sechs Verse dienen dazu, den Kontext näher zu bestimmen und die im letzten Vers zu beobachtende Subtilität nachzuvollziehen. Das Partizip „geheiliget“ wird hier mit „hallowed“ übersetzt. Aber der Übersetzer fügt hinzu: ,as angels demand“. Die Heiligung wird also nicht vom Göttlichen, sondern von Engeln verwirklicht, was eigentlich Hölderlins Ungesagtes schon interpretiert. Diese Interpretation lässt sich wahrscheinlich als pädagogische Geste zugunsten des Inhalts der Elegien erklären, der Hamburger in seinen Essays über die deutsche Dichtung primär interessiert: „Hölderlin's great elegies, written between 1799 and 1801, form a transition from the tragic to the prophetic mode." (Hamburger 1957, 29)

Nick Hoff seinerseits übersetzt Hölderlins Elegien in einem noch eindeutigeren Stil. In „Stuttgart“ steht der Vers: „Aber der Meister pflügt die Mitte des Landes“, und Nick Hoff schreibt: „But the master, the River Neckar, plows down the center" (Hoff 2008, 124-125), wobei er die Bedeutung des Flusses Neckar deutlich betont. Um die Rolle der Dichter zur Geltung zu bringen, expliziert der Übersetzer manchmal Hölderlins Verse mit einer Anpassung der Interpunktion, wie beispielsweise in „Brot und Wein“: „Darum singen sie auch mit Ernst die Sänger den Weingott“ - „That's why they, serious, sing of the wine god, the singers“ (Hoff 2008, 140-141) [meine Hervorhebung, T.B.]. Die Einführung des Kommas vor dem Ausdruck „the singers“ expliziert die Epanalepse von Hölderlin, die ohne Komma rätselhaft und zweideutig anmutet. Nur lässt sich die Einführung des Kommas dadurch rechtfertigen, dass dessen Gebrauch zur Zeit Hölderlins nicht so klar bestimmt war wie heute und seine Abwesenheit wahrscheinlich nicht zur Verrätselung dienen sollte. Hölderlins phantasievolle Interpunktion folgt nicht einer intendierten Ästhetik wie bei Stefan George.

In die zweite Fassung derselben Elegie leitet Hoff im Gegenteil eine Epanalepse ein, um den verzögerten Prädikatsnominativ hervorzuheben: „Dorther kommt und da lachet verpflanzet, der Gott“ - „From there he comes and laughs transplanted, the god." (Hoff 2008, 154-155) [meine Hervorhebung, T.B.]. So tritt die Rolle des Weingotts deutlicher hervor. Die Explizierung der Verse bleibt trotz alledem problematisch, insofern als sie den chaotischen Satzbau Pindars, von dem sich Hölderlin wie schon betont inspirieren lässt, sozusagen zu ordnen droht. Hierin bestehen die Grenzen der schweizerischen und englischen Übersetzungsschule, während die wörtliche Übersetzungsschule der französischen Heideggerianer zur Wiedergabe des Pindarschen Stils beitragen soll. Das Übersetzen setzt eine Wahl voraus, die das bekannte Motto traduttore traditore veranschaulichen kann. 


\section{Die Osmose zwischen den Übersetzungen der Dichter und deren eigener Dichtung}

\subsection{Rouds Vorliebe für Ruhe, Natur und Andacht}

Wir gehen nun zu den Beziehungen zwischen der schöpferischen Übertragung durch Dichter und deren Werk über. Beiderseits des Kanals finden wir Dichter unter den Übersetzern Hölderlins: Roud, Jaccottet und Hamburger. Alle anderen stammen aus dem akademischen Milieu. ${ }^{2}$

Rouds Übersetzung neigt zur Osmose mit dem eigenen Werk. Sie zeugt von seiner Vorliebe für Ruhe, Natur und Andacht. ${ }^{3}$ Bei dieser Osmose handelt es sich jedoch nicht unbedingt um eine bewusste, wie ein Zeugnis von Roud selbst nahelegt:

Des amis qui avaient lu mes poèmes et qui connaissaient des poèmes de poètes allemands que j'ignorais totalement, ont cru surprendre une certaine ressemblance entre ce que j'essayais de faire et l'art de ces poètes [...]. Ma rencontre avec Hölderlin, avec Novalis ont été de cet ordre. ${ }^{4}$ (Roud in Salem 1986, 99-100)

Wir können in einem seiner Texte auch das elegische Harren und ziellose Wandern von Menon: „Täglich geh' ich heraus, und such' ein Anderes immer, / Habe längst sie befragt alle die Pfade des Lands“ (Hölderlin 1992, 267) und das Hölderlinsche Streben nach der verlorenen Einheit der Menschen mit den Göttern, die im Empedokleischen Selbstmord gesucht wird, wiederfinden:

\footnotetext{
L'Attente

[...] Les uns attendent la mort comme une réponse ; d'autres, le temps d'un éclair, sentent en eux-mêmes cette réponse confusément s'ébaucher [...]. Ils s'efforcent vers une unité de l'être, car elle ne pourrait être acquise que par une sorte de suicide [...]. Ils errent çà et là sans autre but que cette attente elle-même. [...] L'écho d'une voix morte leur rend par instant le fantôme d'un frère tout de suite évanoui. (Roud 1989, 248-249)
}

Der Einfluss des elegischen Hölderlin bleibt in diesem Text jedoch nicht ganz unbewusst, wie das folgende Geständnis aus demselben Band suggeriert: „Je vois ce

2 Michel Deguy ausgenommen, der sowohl Professor als auch Dichter ist.

3 Diesen Aspekt entwickelt Ursula Kobiljak: „Often flowers represented objects on which Roud could focus his attention in the following sense: ,Présences véritables, consolantes dans ce no man's land intérieur où je sombrais‘ (J. 394), which is reminiscent of Hölderlin's ,Geh unter, schöne Sonne‘: ,es atmeten / Der dunklen Erde Blüten mich liebend an.' (Kobiljak 1993, 118).

4 Interview von Roud für Radio Suisse Romande von 1966. 
Hoelderlin du temps des hymnes, ayant rompu avec ce que les hommes appellent la ,vie', descendant seul, Diotima morte, Schiller cruellement silencieux, dans sa grande Nuit prophétique.“ (Roud 1989, 195) Im eventuell trauernden Harren, über das Roud hier schreibt, ahnen wir seine Suche bei Hölderlin nach der Lösung eines transzendenten Rätsels im Dasein selbst.

\subsection{Jaccottets Suche nach dem unsagbaren Absoluten}

Was Jaccottet betrifft, so weist Christine Lombez ebenfalls auf eine Osmose hin, die sich wenigstens ab und zu zwischen seinen Übersetzungen und seinem Werk ereignet. Sie erwähnt eine Suche in der Übersetzung selbst nach dem Unerfassbaren, die sich auch in seinem Dichten belegen lässt. Indem er Hölderlin übersetzt, versucht Jaccottet, das Unsagbare zu äußern und den wachenden Dichter als Vermittler zwischen den Sterblichen und dem Göttlichen oder dem Absoluten zu verkörpern, so wie er in der Elegie erscheint. In einem Gedicht mit dem Titel „Le Travail du poète“ [„Die Arbeit des Dichters“] bekennt er sich zu dieser Rolle als Vermittler:

L'ouvrage d'un regard d'heure en heure affaibli n'est pas plus de rêver que de former des pleurs, mais de veiller comme un berger et d'appeler tout ce qui risque de se perdre s'il s'endort. (Jaccottet 1977, 1964)

Im selben Band schreibt er noch:

La nuit n'est pas ce que l'on croit, revers du feu, chute du jour et négation de la lumière, mais subterfuge fait pour nous ouvrir les yeux sur ce qui reste irrévélé tant qu'on l'éclaire. (Jaccottet 1977, 1956)

Hierin erweist sich die Inspiration durch die Elegie „Brot und Wein“ und deren Motiv der Nacht als eindeutig und direkt. Paradoxerweise soll sich die Aufklärung in der finsteren Nacht und im Warten auf das kommende Tageslicht ereignen. Auch im Fall Jaccottets spielt demnach die Suche nach dem Absoluten bzw. Göttlichen eine wichtige Rolle in der Synergie zwischen dem Übersetzen und dem Dichten. 


\title{
3.3 Hamburgers elegische Brandmarkung der antidichterischen Liberalisierung der Welt
}

Bei Hamburger finden wir eine ähnliche Inspiration durch die Elegie „Brot und Wein“; insbesondere im Gedicht „Hölderlin“:

\author{
Hölderlin \\ (Tübingen, December 1842) \\ Diotima is dead, and silent \\ The island's singing bird. \\ The temple I raised from ruin \\ Fallen again.
}
Where is the flame I stoked from ashes
Of the mind? Where are the heroes
And my pulsing song?
Nothing stirs on the lakes of time.
Give back my agony,
O stir the forest's sap,
Sweep my slow blood.
And yet, no caged old panther I,
Pacing my madness. These muttered words
Are gates, not bars, where only I can pass.
This is my wisdom, where no flowers grow,
No weeds, this is my peace.
I am calm now, with the world
Locked out, bowed to the door;
My meandow end is pensioned by the gods.
They did not hear,
O crippled Fate, the grimy idol's
Golden teeth led them away.

I have no tears to mourn forsaken gods

Or my lost voice.

This is my wisdom where no laughter sounds,

No sighs, this is my peace.

Glory is gone, and the swimming clouds;

My dumb hand grips the frozen sky,

A black bare tree in the winter dusk.

(Hamburger 1942, 29)

Hamburger präzisiert zu diesem Gedicht, der Monolog müsse auch als „Persona“ aufgefasst werden, nicht als eine Rekonstruktion des historischen Hölderlin. (Hamburger 1963-1964, 85) Nun sieht Walter Eckel eben in der Elegie „Menons 
Klagen um Diotima“ eine wichtige Anspielungsquelle für dieses Gedicht Hamburgers, das von jenem, durch den Abschied und die Trennung von Diotima verursachten, tiefen Leid („Diotima is dead“) und vom erinnerten Glück Hölderlins handelt. (Vgl. Eckel 1991, 19) Nur stützt sich Hamburger auf das Hölderlinsche Motiv vom Verlust Diotimas, um seinem Gedicht einen elegischen Akzent zu geben und über die Trauer hinaus eine Realität zu denunzieren, die der Dichtung schadet, nämlich die Liberalisierung der Welt (,the grimy idol's / Golden teeth led them away.“). Und um Hamburgers „Verzweiflung über die zum Markt verkommene Welt“ und den damit verbundenen Nachlass vom Interesse an der Lyrik zu begründen, zitiert Eckel Hölderlins berühmte Frage aus „Brot und Wein“: „wozu Dichter in dürftiger Zeit?“ (Hölderlin 1992, 290). In einem Brief an Eckel, der ihn nach einer Erläuterung fragte, legte Hamburger selbst letztere Verse aus, die die Liberalisierung der Welt brandmarken: „Bei grimy idol dachte ich wahrscheinlich an das frühe Industriezeitalter und den Frühkapitalismus.“ (Eckel 1991, 196) Die Kritik am Kapitalismus in der Dichtung erweist sich zwar nicht als besonders originell, der Leser sieht aber wohl ein, dass der Übersetzer Hamburger in der eigenen Dichtung Hölderlins Elegie als intertextuelle Vorlage und Inspirationsquelle benutzen kann.

Es lässt sich daraus folgern, dass wir nun einmal bei den übersetzenden Dichtern eine richtige Osmose zwischen dem übersetzten Hölderlin und dem eigenen Werk bestätigen können. Die Intertextualität betrifft demnach sowohl das Thema der Trauer um einen lieben Mitmenschen, als auch das der bedeutenden sozialen Funktion des Dichters. Die Bedeutung einer solchen Intertextualität liegt wahrscheinlich an der Stärke der von diesen Themen hervorgerufenen Gefühle, die einen beiderseits des Kanals rühren.

Abschließend ließe sich feststellen, dass die wörtliche Übersetzung philosophischer Inspiration zwar darauf zielt, alle dichterischen und philosophischen Besonderheiten des deutschen Textes wiederzugeben. Ein solches Vorgehen setzt aber vor allem in einer dem Deutschen so entfernten Sprache wie dem Französischen Inkorrektheiten voraus, die der deutsche Text nicht aufweist und die die Grenzen einer solchen Übersetzungsmethode aufzeigen. Um den „Dichter der Dichter“ (Heidegger) zu übersetzen, scheinen Dichter-Übersetzer trotz ihrer vielleicht hie und da übertriebenen Interpretationsanstrengungen und Explizierungen am besten geeignet, was aufgrund der verwandten Inspiration ein Hin- und Her-Pendeln zwischen dem übersetzten Text und dem eigenen Werk mit sich bringen kann. Es handelt sich zwar um keinen originellen und neuen Modus des Übersetzens, der hier als Nachdichtung aufgefasst wird. Ich habe jedoch im Rahmen dieser Studie am Beispiel Hölderlins die Vorteile einer solchen Übersetzungsmethode aufzuzeigen versucht. In diesem Sinne definiert Antoine Berman die dichterische Dynamik der Übersetzung: „La traduction mériterait son sécu- 
laire statut ancillaire si elle ne devenait pas enfin un acte de décentrement créateur conscient de lui-même.“ (Berman 1984, 40)

\section{Literaturverzeichnis}

Berman, Antoine. L'épreuve de l'étranger. Paris: Gallimard, 1984.

Chevrel, Yves, und Jean-Yves Masson. Histoire des traductions en langue française. Paris:

Verdier, 2014.

Courtine, Jean-François. Hölderlin. Paris: L'Herne, 1989.

Eckel, Walter. Von Berlin nach Suffolk zur Lyrik Michael Hamburgers. Würzburg: Könighausen \& Neumann, 1991.

Fédier, François. Hölderlin Pain et vin. La Clayette: M. Chandeigne, 1983.

Fédier, François. Douze poèmes. Giromagny: Orphée, La Différence, 1989.

Hamburger, Michael. „Englische Hölderlin-Gedichte“. Hölderlin-Jahrbuch 13 (1963-1964): 80-103.

Hamburger, Michael. Friedrich Hölderlin. Selected Poems and Fragments. London: Penguin Books, 1998 (1966).

Hamburger, Michael. Oxford and Cambridge Writing. Oxford: Oxford University Press, 1942.

Hamburger, Michael. Hölderlin: His Poems Translated, with a Critical Study of the Poet. London: Harvill Press, 1952.

Hamburger, Michael. Reason and Energy. London: Routledge \& Kegan Paul, 1957.

Heidegger, Martin, Erläuterungen zu Hölderlins Dichtung. Frankfurt/M.: Vittorio Klostermann, 1971 (1944).

Hoff, Nick. Odes and Elegies - Friedrich Hölderlin. Middletown: Weysleyan University Press, 2008.

Hölderlin, Friedrich. Sämtliche Werke, Gedichte, Bd. 1. Frankfurt/M.: Deutscher Klassiker Verlag, 1992.

Jaccottet, Philippe. Hölderlin, Euvres. Paris: Gallimard, 1967.

Jaccottet, Philippe. Poésie 1946-1967. Paris: Gallimard, 1977.

Jaccottet, Philippe. Une Transaction secrète. Paris: Gallimard, 1987.

Kobiljak, Ursula. Echoes of Germanic Poetry in the Work of Gustave Roud. New York: American University Studies, Peter Lang, 1993.

Lombez, Christine. Transactions secrètes Philippe Jaccottet traducteur de Rilke et de Hölderlin. Arras: Artois Presse université, 2003.

Lombez, Christine. „Entre lecture et écriture : Philippe Jaccottet ,passeur de Hölderlin““. Poésie 2004100 (2004): 20-24.

Maggiori, Robert. "La «forêt obscure» de Heidegger.“ Libération 4 November 2013. https://next. liberation.fr/livres/2013/11/06/la-foret-obscure-de-heidegger_945105 (28. Juni 2018).

Roud, Gustave. Poèmes de Hölderlin. Lausanne: Mermod, 1942.

Roud, Gustave. Écrits, vol. II. Lausanne: Bibliothèque des arts, 1989.

Salem, Gilbert. Gustave Roud - Qui suis-je? Lyon: La Manufacture, 1986.

Schmidt, Jochen. „Pindar als Genie-Paradigma im 18. Jahrhundert“. Goethe-Jahrbuch 101 (1984): 63-73. 
Thomas Buffet promovierte 2011 über die Erneuerung der elegischen Gattung durch André Chénier und Friedrich Hölderlin. Er lehrt heute deutsche Literatur und Übersetzung in den Pariser Vorbereitungsklassen und Komparatistik an der Sorbonne. Seine Forschungsarbeiten untersuchen die europäische Romantik und die Geschichte der Übersetzung. 